\title{
Pathogenesis of tears of the retinal pigment epithelium*
}

\section{J. DONALD M. GASS}

From the Department of Ophthalmology, Bascom Palmer Eye Institute, University of Miami Medical School, Miami, Florida, USA

SUMMARY This report confirms a previous report that elderly patients with serous detachments of the pigment epithelium prior to and after developing a pigment epithelial tear at one border present a characteristic ophthalmoscopic and fluorescein angiographic appearance. Evidence is presented that subpigment epithelial choroidal neovascularisation and not irregular separation of the basement membrane from its pigment epithelium is the primary cause of the detachment and tear in the pigment epithelium.

In 1981 Hoskin et al. described the ophthalmoscopic and fluorescein angiographic features of (1) serous detachments of the pigment epithelium that were prone to develop a tear along their margin, and (2) the characteristic post-tear appearance of these lesions which occur in patients with senile macular degeneration.' They postulated that irregular separation of the pigment epithelium from its basement membrane was responsible for the angiographic picture and for determining the site of the pigment epithelial tear. The purpose of this presentation is to confirm their clinical observations and to suggest an alternative explanation for the funduscopic and angiographic changes as well as the pathogenesis of pigment epithelial tears.

\section{Case reports}

CASE 1

An 85-year-old man developed metamorphopsia in his right eye: He had lost central vision in his left eye previously because of senile disciform macular degeneration. His visual acuity was $20 / 30$ in the right eye and finger counting in the left eye. In the right macula there was a dome-shaped area of detachment of the pigment epithelium (Fig. 1A). The temporal half of the pigment epithelial detachment was slightly more elevated than the nasal portion, where small

* Presented at the International Symposium on Fluorescein Angiography, Coronado, California, USA, 24-30 October, 1982.

Correspondence to J. Donald M. Gass, MD, Bascom Palmer Eye Institutc, PO Box 016880, Miami, Florida 33101, USA. drusen were present. There was no evidence of subretinal neovascularisation biomicroscopically. Fluorescein angiography revealed delayed and incomplete fluorescein staining of the nasal part of the pigment epithelial detachment (Fig. 1B). This was interpreted as evidence of the presence of fibrovascular tissue and blood pigment lying beneath the nasal part of the pigment epithelial detachment. He returned five weeks later because of further reduction

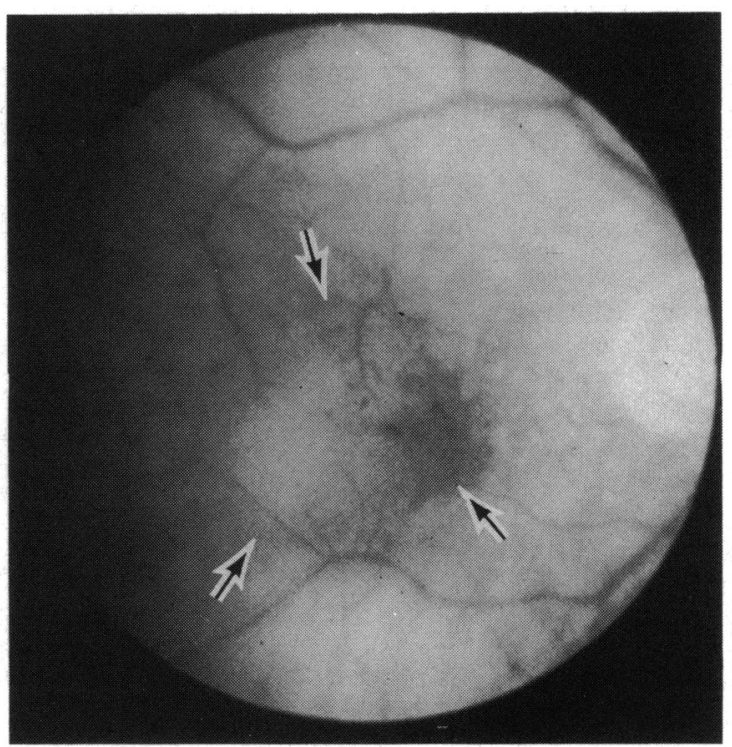

Fig. 1A Case 1.25 September 1968. Pigment epithelial detachment (arrows). 


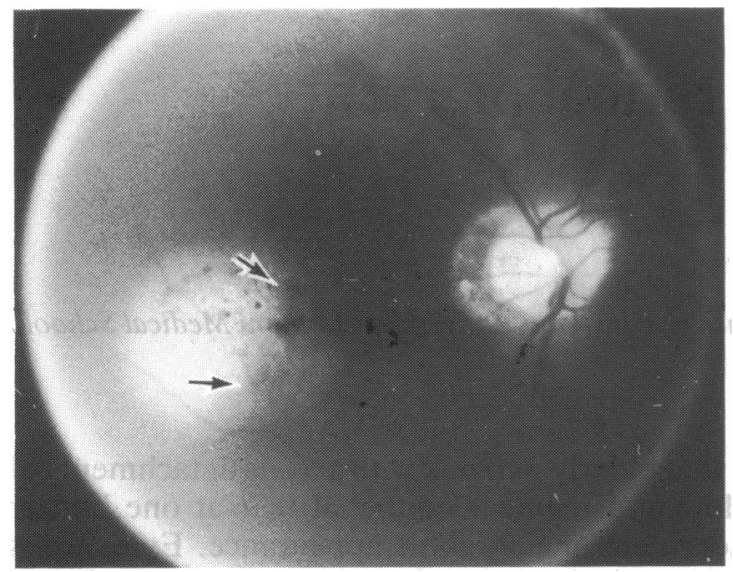

Fig. 1B Case 1. Note incomplete staining of subpigment epithelial exudate nasally (arrows).

of visual acuity to $5 / 200$. The temporal two-thirds of the previous pigment epithelial detachment was replaced by a sharply defined zone of what was interpreted as atrophy of the pigment epithelium and the nasal one-third by a solid-appearing pigmented mound (Fig. 1C). Fluorescein angiography revealed marked hyperfluorescence consistent with either marked attenuation or absence of the pigment epithelium temporally (Fig. 1D). There was irregular hyperfluorescence and late staining of the surface of the pigmented mound of tissue nasally (Figs. 1D, E). Two months later there was subretinal blood around the margins of the pigmented mound (Fig. 1F). One year later a large choroidal neovascular membrane extended throughout the centre of the macula.

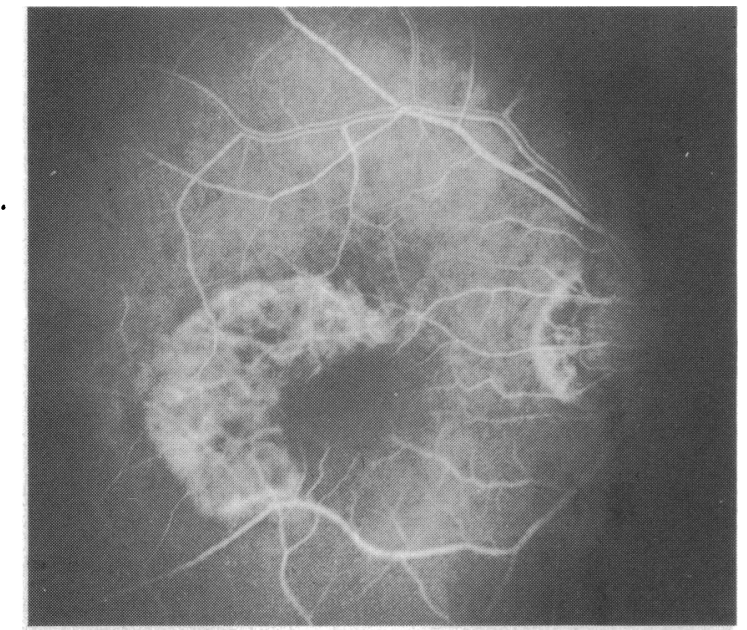

Fig. 1D

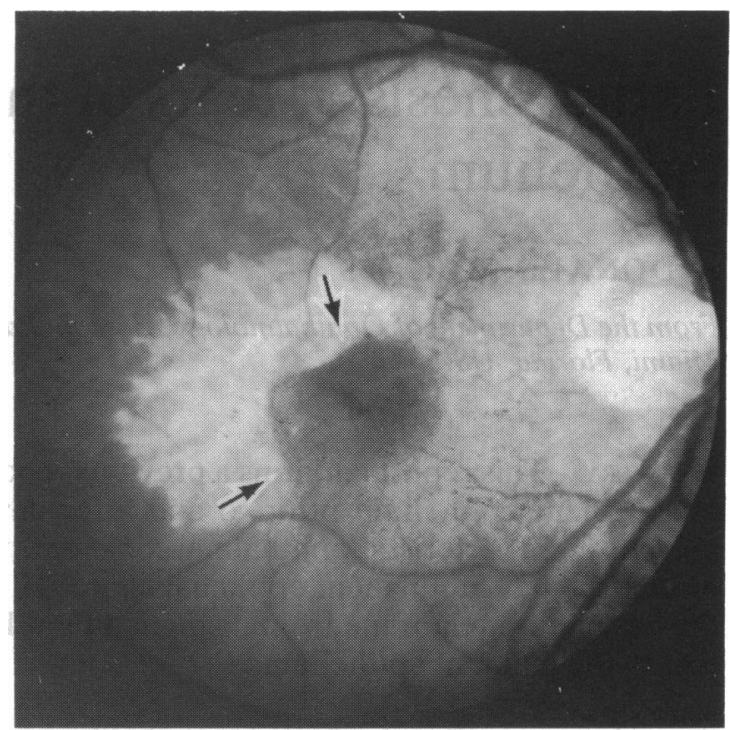

Fig. 1C Case 1.23 October 1968. Pigmented mound (arrows) and zone of atrophic or missing pigment epithelium indicative of a previous tear in the pigment epithelium.

Comment. Colour stereophotographs of the preand post-tear appearance of this fundus lesion were previously published and interpreted as showing geographic atrophy of the pigment epithelium caused by spontaneous reattachment of atrophic thinned pigment epithelium adjacent to a zone of the subpigment epithelial fibrovascular proliferation. ${ }^{23}$ I believe now, however, that Hoskin et al. are correct in assuming that this fundus change is usually caused

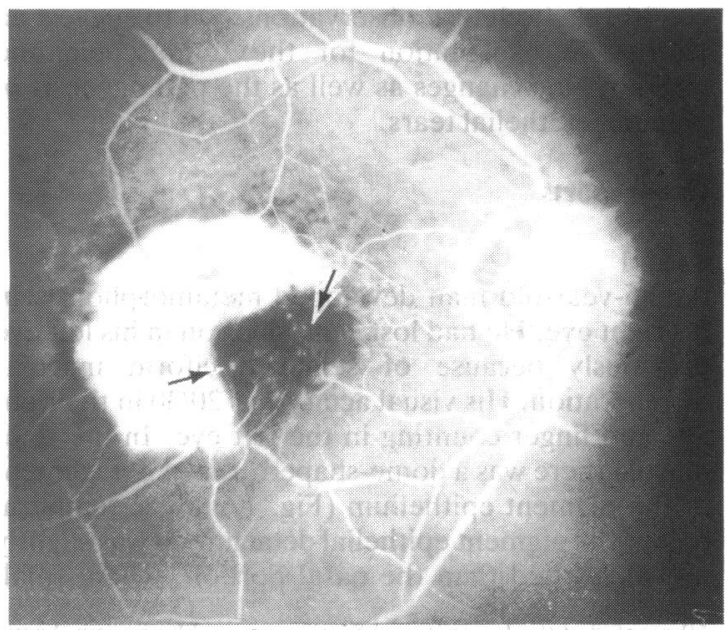

Fig. 1E

Figs. 1D and E Case 1. Angiogram showing pinpoint areas of fluorescence (arrows) and late staining indicative of blood vessels within the pigmented mound. 


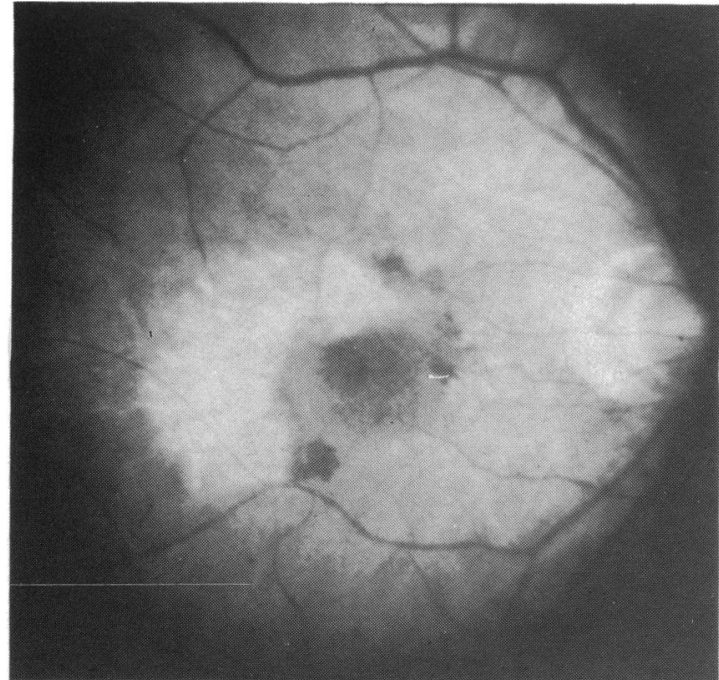

Fig. 1F Case 1.19 December 1968. Notesubretinalblood at edge of pigmented mound.

by a tear in the pigment epithelium. I still, however, interpret the pre- and post-tear angiogram as indicating the presence of fibrovascular tissue with or without blood pigment in the nasal portion of the detachment. In this case this interpretation was sustained by the subsequent development of subretinal blood and angiographic evidence of unequivocal choroidal neovascularisation. If the pigmented mound of tissue nasally was composed solely of rolled-up and

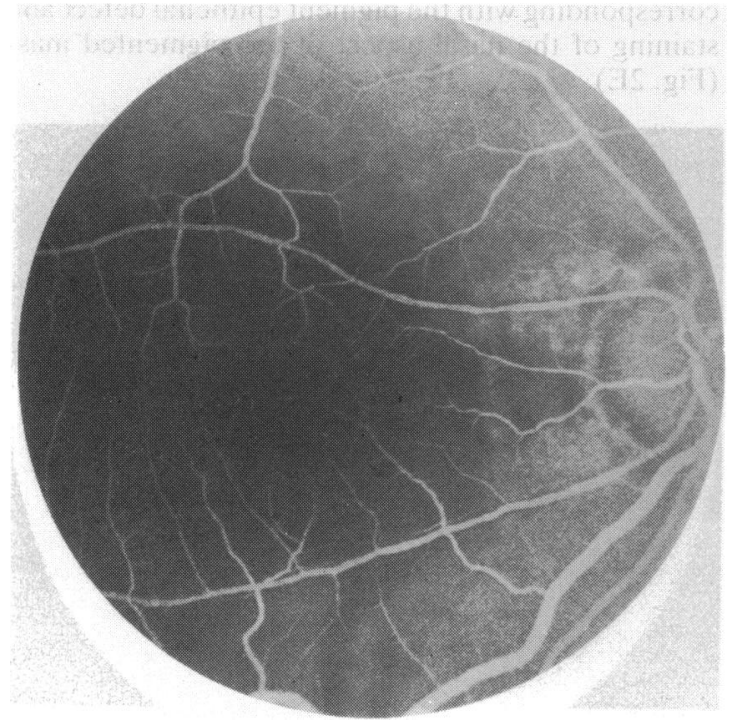

Fig. 2B

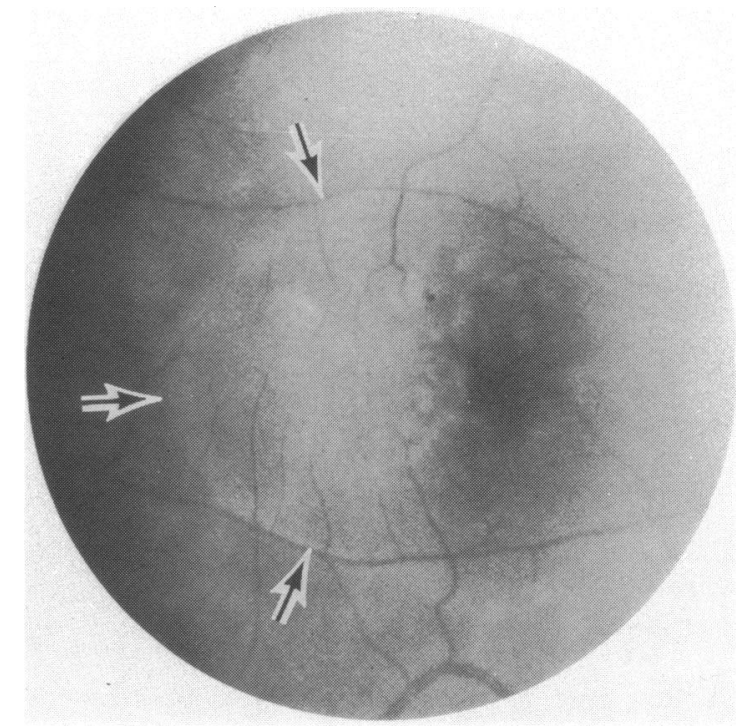

Fig. 2A Case 2.7July 1979. Serous detachment of the pigment epithelium (arrows).

retracted pigment epithelium, it should remain nonfluorescent throughout angiography.

\section{CASE 2}

A 76-year-old man complained of metamorphopsia in the right eye of four weeks' duration. His visual acuity was $20 / 200$ in the right eye. He had a mild cataract and a large serous detachment of the pigment

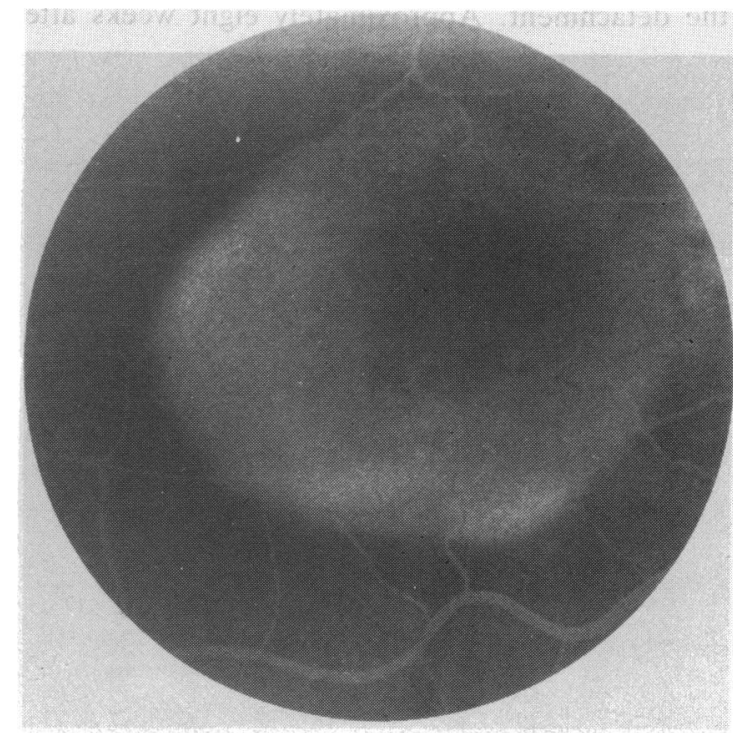

Fig. 2C

Figs. 2B and C Case 2. Delayed and incomplete staining of subretinal exudate. 


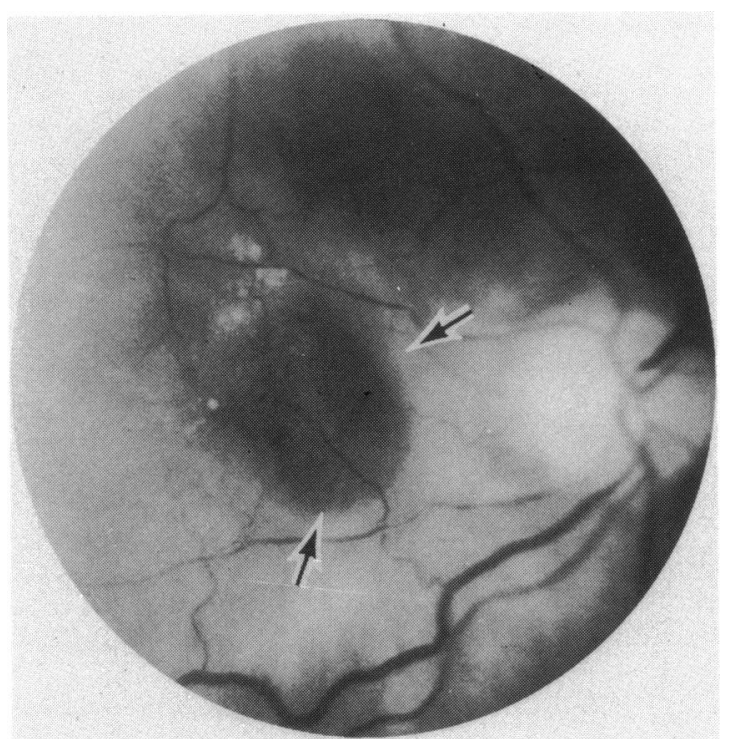

Fig. 2D Case 2. 12 October 1970. Post-tear appearance with pigmented mound (arrows).

epithelium in the right macula (Fig. 2A), and multiple drusen in the left macula. Angiography confirmed the presence of the detachment and revealed a delay in the overall fluorescein staining and incomplete staining of the nasal part of the detachment (Figs. 2B, C). Argon laser photocoagulation was placed along the margins of the detachment. Five weeks later the detachment was unchanged. There was a small amount of subretinal blood at the inferior margin of the detachment. Approximately eight weeks after

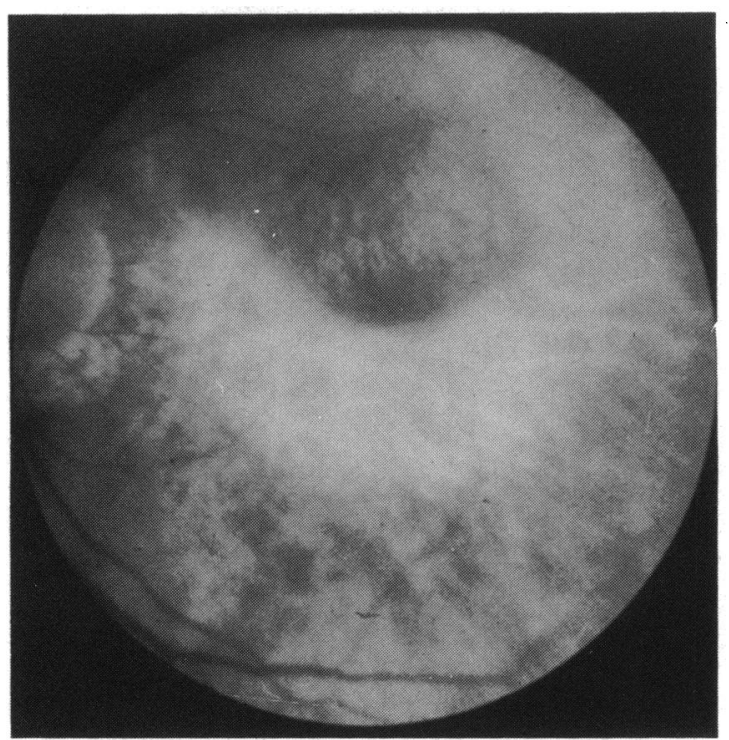

Fig. 3A Case 3. Postpigment epithelial tear.

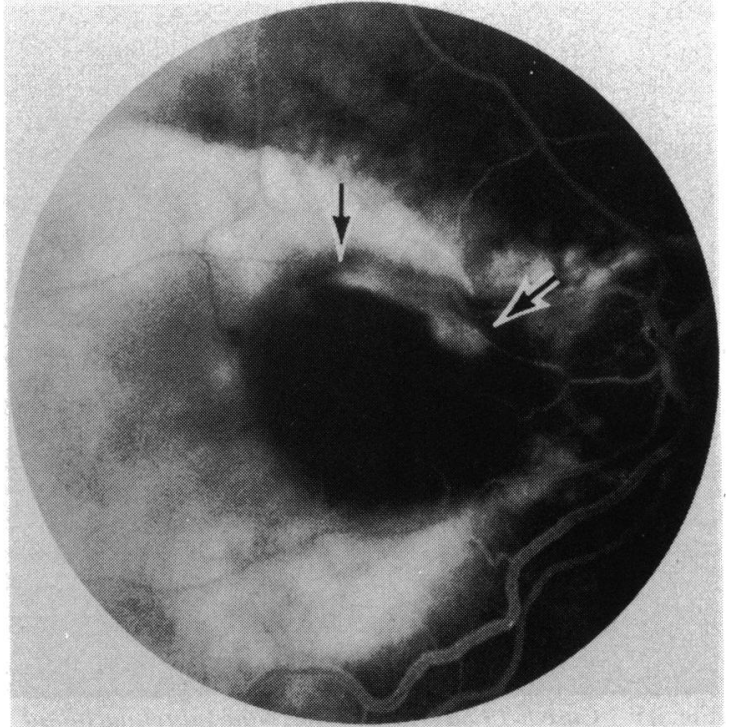

Fig. 2E Case 2. Angiogram showing mottled fluorescein staining of surface of pigmented mound (arrows).

treatment he noted a worsening of the vision in the right eye. Ten weeks after laser treatment the pigment epithelial detachment was replaced by a large pigment epithelial defect temporally and an elevated pigmented mass nasally (Fig. 2D). There was a serous detachment of the retina overlying the pigment epithelial defect. Circinate exudate was present at the temporal margin of the pigmented mass. Angiography revealed marked hyperfluorescence corresponding with the pigment epithelial defect and staining of the nasal aspect of the pigmented mass (Fig. 2E).

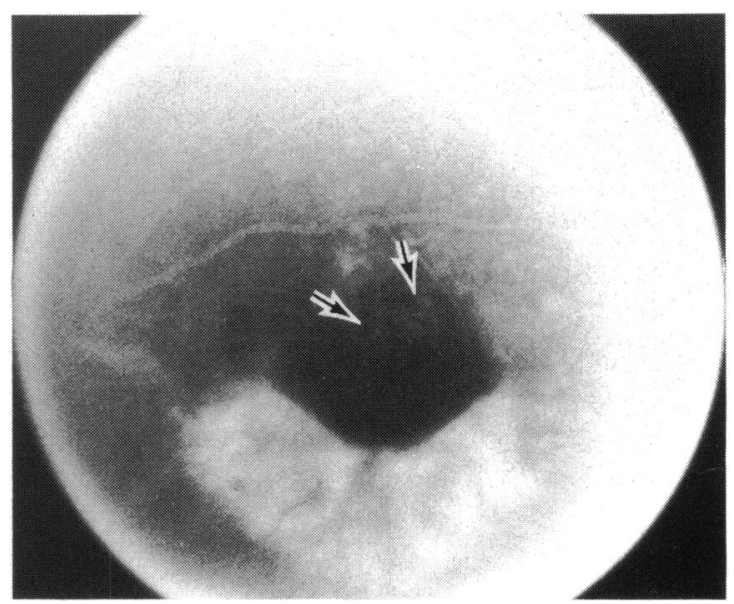

Fig. 3B Case 3. Note faint areas of staining (arrows), indicative of blood vessels within the subretinal pigmented mass. 


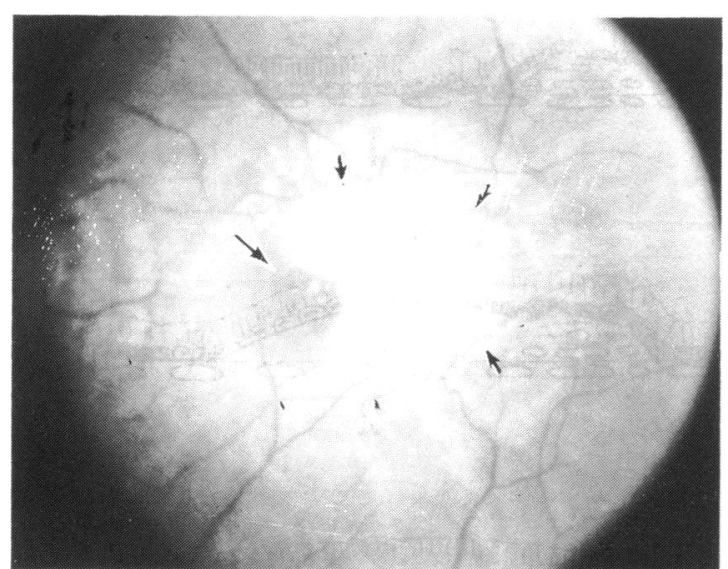

Fig. 4A Postpigment epithelial tear. Note subretinal pigmented mound (large arrow), edge of torn pigment epithelium (small arrows) and surrounding subretinal precipitates in area of resolving detachment.

Comment. Between five and 10 weeks after argon laser treatment the patient probably developed a tear along the temporal margin of the pigment epithelial detachment. The subretinal blood noted just prior to the tear and the circinate exudate afterwards are evidence that choroidal neovascularisation was present at the time of the tear.

\section{CASE 3}

An 80-year-old man consulted his local ophthalmologist because of recent loss of vision in the right eye. He had lost central vision in the left eye one year previously. His physician noted serous macular detachment in the right eye and a pigmented mass that he suspected might be a melanoma in the left macula. Examination at the Bascom Palmer Eye Institute revealed bilateral senile macular disease and a left fundus picture typical of that following a tear in the pigment epithelium (Fig. 3A). There was faint mottled fluorescein staining on the surface of the pigmented mound (Fig. 3B).

\section{CASE 4}

A 68-year-old man noted an abrupt loss of central vision in the left eye six weeks prior to examination. Visual acuity was 20/25 in the right eye, and 10/200 in the left eye. He had drusen in the right macula and in the left eye had ophthalmoscopic and angiographic evidence of having previously developed a pigment epithelial tear (Figs. 4A, B).

\section{Discussion}

Hoskin et al. described the following three features of the pretear appearance of the pigment epithelial

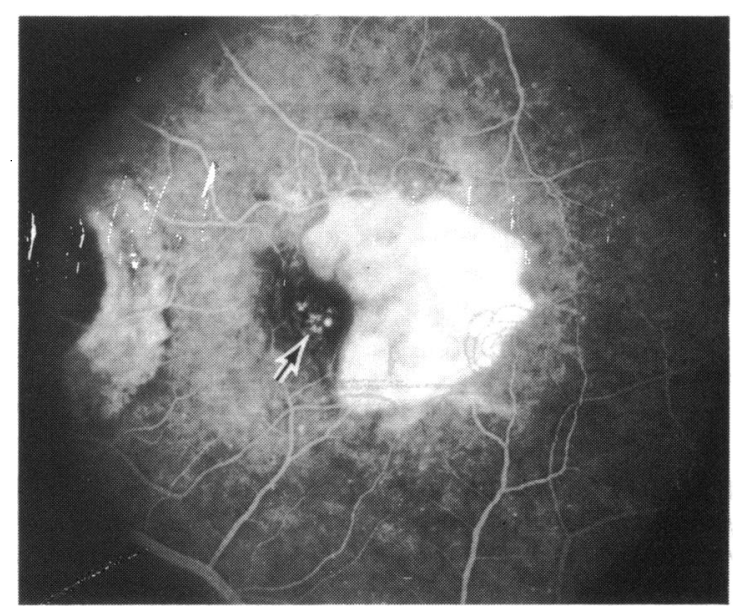

Fig. 4B Staining (arrow) indicative of blood vessels within the mound.

detachments which occurred in 8 of 12 patients observed prior to development of a tear: (1) uneveness of drusen distribution in the area of the pigment epithelial detachment; (2) uneven hyperfluorescence in the area of most drusen; (3) greater elevation and more uniform hyperfluorescence of the drusenfree area of the detachment.' In four eyes the late fluorescein staining was uniform. The authors described the following features of the post-tear phase of the lesion: (1) a zone of absent pigment epithelium caused by a tear occurring at the edge of that portion of the pigment epithelial detachment that was uniformly fluorescent; (2) a pigmented mound, which they attributed solely to retracted and rolled-up pigment epithelium at one area of the bared area of Bruch's membrane; (3) non-fluorescence of the pigmented mound; and (4) no evidence of subretinal neovascularisation in spite of their observation of subretinal blood in five of 44 eyes.

Fig. 5 is a diagram depicting their explanation for the pre- and post-tear appearance of these lesions. They suggest that detachment of the pigment epithelium from its underlying basement membrane in the area of greatest elevation is responsible for the more uniform area of hyperfluorescence and for making the pigment epithelium more susceptible to tearing.

My observations of the pretear funduscopic and angiographic appearance (Figs. 1 and 2) coincide with that of Hoskin et al., ' although my interpretation of these findings is different. So far I have not seen a tear develop in a pigment epithelial detachment that showed early uniform fluorescein staining. As regards the post-tear lesion, I concur with their first two observations but not the last two. In my experience 


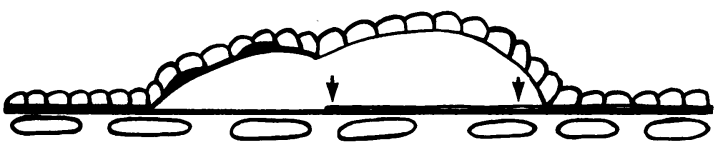

PRE-IEAR

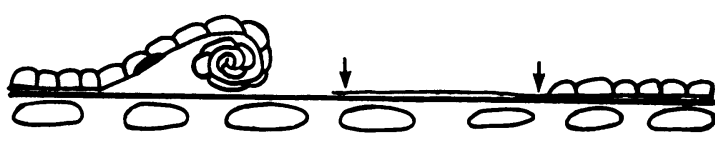

POST-IEAR

Fig. 5 Diagram of Hoskin et al.'s theory of cause of pigment epithelial tear. Arrows indicate portion of basement membrane detached from its overlying pigment epithelium.

angiography of the post-tear lesions has shown streaks or spots of early fluorescence and small areas of staining at the surface of the pigmented mound in most cases (Figs. 1, 2, 3, and 4). I interpret these findings, as well as the delayed and non-uniform staining of the pre-tear lesions, as angiographic evidence of probable choroidal neovascularisation. Since the mound of fibrovascular tissue is partly covered by the retracted and rolled-under pigment epithelium, in some cases neither early nor late fluorescence will be evident.

The hypothesis that separation of the pigment epithelium from its basement membrane is responsible for the uniform fluorescence and for predisposing the pigment epithelium to tear is not supported by reported light microscopic, electron microscopic, or clinical findings. The basement membrane is tightly adherent to the pigment epithelium, much more so than to the collagenous portion of Bruch's membrane.$^{4}$ It is likely, therefore, that detachment of the basement membrane from its pigment epithelium rarely, if ever, occurs. Electron microscopic studies have shown that in the case of drusen the basement membrane remains attached to the elevated pigment epithelium. ${ }^{5}$ In the case of one large serous detachment of the pigment epithelium in an eye enucleated because of suspected melanoma in an elderly woman, PAS stains revealed evidence that the basement membrane, as well as multiple small drusen, was detached along with the pigment epithelium. ${ }^{6}$ Clinically, serous detachments of the pigment epithelium occurring in young as well as older patients characteristically show early uniform staining with fluorescein, which, if the authors were correct, would suggest that in most cases detached pigment epithelium is separated from its basement membrane, a finding that to my knowledge has never been demonstrated.

Fig. 6 depicts what I believe is a more plausible
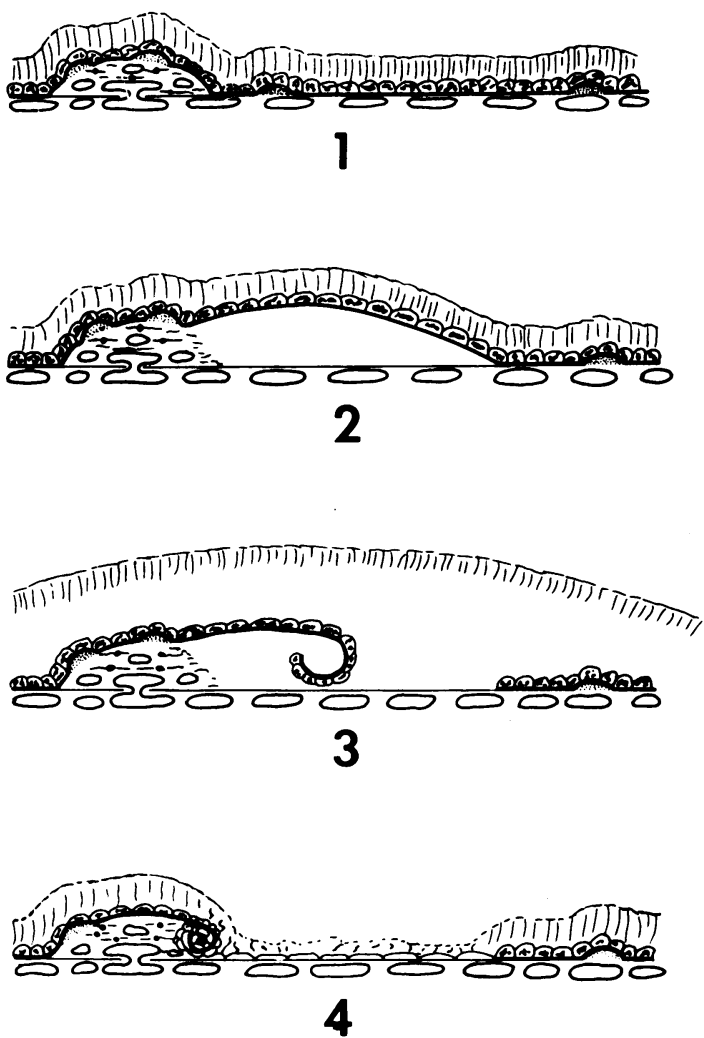

Fig. 6 Diagram of stages of development of organised serous detachment of pigment epithelium and pigment epithelial tear. Stage 1: Occult choroidal neovascularisation. Stage 2: Secondary serous detachment of pigment epithelium and its basement membrane. Stage 3: Acute tear of pigment epithelium and secondary retinal detachment. Stage 4: Posttear regrowth of hypopigmented pigment epithelium and reattachment of retina.

hypothesis to explain the pathogenesis of spontaneous pigment epithelial tears. In stage 1 new vessels invade and grow through Bruch's membrane into the subpigment epithelial space. Over a period of weeks or months these vessels, together with fibrocytes, proliferate to form a progressively enlarging fibrovascular mound that gradually elevates the basement membrane, drusen, pigment epithelium, and retina. Except for the elevation this may cause minimal or no changes in the ophthalmoscopic, angiographic, or histological appearance of the overlying retina and pigment epithelium, and it may produce no symptoms. A rapid change in permeability of the new vessels within this occult lesion produces a serous detachment of the adjacent pigment epithelium (stage 2 ). This detachment is typically slightly broader and more elevated than the area of organised detachment. The normal granularity of the pigment epithelium 
and the drusen in the area of serous detachment typically disappear from biomicroscopic view. The drusen presumably dissolve within the serous exudate. The pigment epithelium remains anchored by its attachment to one side of the organised mound. As the pressure of escaping serous fluid detaches the pigment epithelium away from the opposite side of the mound, a point is reached where the pigment epithelial cells cannot withstand the pressure required to separate it from Bruch's membrane, and a tear occurs at the junction of the attached and detached pigment epithelium (stage 3, Fig. 6). Serous fluid moves from beneath the pigment epithelium into the subretinal space as the free edge of the tear retracts in an accordion-like fashion and rolls under toward the organized mound. A localised serous detachment of the retina larger than the area of detached pigment epithelium persists until the pigment epithelial cells along the margin of the tear slide and proliferate to cover the defect with hypopigmented cells. We seldom have an opportunity to see stage 3 , since it probably lasts only a matter of days or a few weeks. Once re-epithelialisation occurs, the subretinal exudate disappears (stage 4, Fig. 6). In some cases the defect is largely covered with a semitransparent grey tissue that probably represents fibrous metaplastic pigment epithelium.

Uneven elevation of the pigment epithelial detachment with preservation of the near normal granularity of the pigment epithelium and small drusen in the less elevated organised portion of the detachment, the incomplete and uneven staining of the area of the organisation, and the delay in staining of the adjacent subpigment epithelial exudate, probably caused by its high protein and blood pigment content, are three of the clues nearly always present in patients with detachment destined to develop a tear in the pigment epithelium.

If the above hypothesis is correct, it has important therapeutic implications. Any attempt to flatten a partly organised pigment epithelial detachment by photocoagulation along the margin of the detachment ${ }^{7-9}$ will not only fail in flattening the area of detachment but may increase the risk of a tear. The use of a grid pattern of laser to the surface of the organised portion of the detachment " " may increase the permeability of the new vessels, the subpigment epithelial hydrostatic pressure, and the risk of a tear. Conversely, if that portion of the pre-tear lesion that stains incompletely lies outside the foveal avascular zone, moderately intense laser treatment to the entire area of neovascular complex may be successful in flattening the adjacent areas of serous detachment of the pigment epithelium.

Varying results of photocoagulation of serous detachment of the pigment epithelium have been reported..$^{8-13}$ One of the reasons for this variability has been the lack of uniformity in the recognition of the many subtle clues to the presence of new vessels beneath the pigment epithelium. ${ }^{14-16}$

Two recent reports have also suggested the unlikelihood that detachment of the pigment epithelium from its basement membrane is the cause of pigment epithelial tears. ${ }^{1718}$ One report noted evidence of choroidal neovascularisation in five of six patients with a tear. The others noted subretinal and subpigment epithelial blood following development of a large tear with a visible rolled margin; however, this was attributed to mechanical disruption of the choriocapillaris at the site of the tear. ${ }^{18}$

This investigation was supported in part by Public Health Service Research Grant 5R10 EY02549, Department of Health and Human Services, National Institutes of Health, National Eye Institute, Bethesda, Maryland.

\section{References}

1 Hoskin A, Bird AC, Schmi K. Tears of detached retinal pigment epithelium. Br J Ophthalmol 1981; 65: 417-22.

2 Gass JDM. Stereoscopic atlas of macular diseases. St Louis: Mosby, 1970: 39.

3 Gass JDM. Stereoscopic atlas of macular diseases. 2nd ed. St Louis: Mosby, 1977: 73.

4 Goldbaum MH, Madden K. A new perspective on Bruch's membrane and the retinal pigment epithelium. BrJ Ophthalmol 1982; 66: 17-25.

5 Hogan MJ, Alvarado J. Studies of the human macula. IV. Aging changes in Bruch's membrane. Arch Ophthalmol 1967; 77: 41020.

6 Gass JDM. Pathogenesis of disciform detachment of the neuroepithelium. III. Senile disciform macular degeneration. Am J Ophthalmol 1967; 63: 617-44.

7 Gass JDM. Stereoscopic atlas of macular diseases. 2nd ed. St Louis: Mosby, 1977: 390-1.

8 Jepson CN, Wetzig PC. Photocoagulation in disciform macular degencration. Am J Ophthalmol 1969; 67: 920-30.

9 Gass JDM. Drusen and disciform macular detachment and degeneration. Arch Ophthalmol 1973; 90: 206-17.

10 Bird AC. Treatment of senile macular degeneration by photocoagulation. Br J Ophthalmol 1974: 58: 367-76.

11 The Moorfields Macular Study Group. Retinal pigment epithelial detachments in the elderly: a controlled trial of argon laser photocoagulation. BrJ Ophthalmol 1982; 66: 1-16.

12 Braunstein RA, Gass JDM. Serous detachments of the retinal pigment epithelium in patients with senile macular disease. $A m J$ Ophthalmol 1979; 88: 652-60.

13 Meredith TA, Braley RE, Aaberg TM. Natural history of serous detachments of the pigment epithelium. Am J Ophthalmol 1979; 88: $643-51$.

14 Gass JDM. Stereoscopic atlas of macular diseases. 2nd ed. St Louis: Mosby, 1977: 392.

15 Gass JDM. Radial chorioretinal folds. A sign of choroidal neovascularization. Arch Ophthalmol 1981; 99: 1016-8.

16 Gass JDM. Serous retinal pigment epithelial detachment with a 'notch': a sign of choroidal neovascularization. Retina in press.

17 Cantrill HL, Ramsay RC, Knobloch WH. Rips in the pigment epithelium. Arch Ophthalmol 1983; 101: 1074-9.

18 Green SW, Yarian D. Acute tear of the retinal pigment epithelium. Retina 1983; 3: 16-20. 